\title{
Germanica
}

\section{Alles geht? Beliebigkeit und Form in der deutschen Lyrik der achtziger Jahre. Ein Vortrag}

Tout marche? Absence de critères et forme dans le lyrisme des années quatrevingt.

Helmut Arntzen

\section{(2) OpenEdition}

Journals

Édition électronique

URL : http://journals.openedition.org/germanica/1465

DOI : 10.4000/germanica. 1465

ISSN : 2107-0784

\section{Éditeur}

Université de Lille

\section{Édition imprimée}

Date de publication : 30 décembre 1997

Pagination : 13-31

ISBN : 977098426320321

ISSN : 0984-2632

Référence électronique

Helmut Arntzen, « Alles geht? Beliebigkeit und Form in der deutschen Lyrik der achtziger Jahre. Ein Vortrag », Germanica [En ligne], 21 | 1997, mis en ligne le 05 juin 2012, consulté le 06 octobre 2020. URL : http://journals.openedition.org/germanica/1465; DOI : https://doi.org/10.4000/germanica.1465

Ce document a été généré automatiquement le 6 octobre 2020.

(c) Tous droits réservés 


\section{Alles geht ? Beliebigkeit und Form in der deutschen Lyrik der achtziger Jahre. Ein Vortrag}

Tout marche? Absence de critères et forme dans le lyrisme des années quatrevingt.

Helmut Arntzen

1 Was ist das Produktive an den vier großen Perioden deutscher Lyrik: Minnesang, Barock, Goethezeit und Romantik, Jahrhundertwende und Expressionismus? Neue Themen, neue Formen? Die Liebe und der Tod, Landschaft und Jahreszeit erhalten sich als Themen über Jahrhunderte hinweg; Sonett, Volksliedstrophe, jambischer und trochäischer Vierheber, Reim tun es auch. Sicher, es kommt für eine Zeitlang etwas hinzu und schwindet wieder: Geistliches, Politisches, Kunst im Themenregister; Alexandriner, antike Formen, romanische, Hymne. Aber nicht das macht die Lyrik bedeutend. Die Sturm-und-Drang-Hymne Goethes war sicher in ihrer Zeit eine neue und ungewohnte Form, doch vorher, daneben und nachher kennen wir von Goethe immer wieder Lieder, wir kennen Stanzen, wir kennen Sonette und viele andere traditionelle Formen. Wir haben ein Gedicht wie Selige Sehnsucht mit einem vergleichsweise ungewohnten Thema, aber daneben auch im Westöstlichen Divan zahlreiche Liebesgedichte. Will sagen: bis ins 20. Jahrhundert hinein gibt es keine Anzeichen dafür, daß das Aparte oder Triviale eines Themas, daß die Eigentümlichkeit oder die Geläufigkeit der Form darüber entscheiden, ob ein Gedicht wichtig oder irrelevant sei. Sichtlich geht es darum, wie das Traditionelle aufgenommen und verarbeitet, wie das Originelle mit der lyrischen Tradition verbunden werde, wie es sich mit ihr auseinandersetze. Wo das eine oder das andere gar nicht mehr geschieht, entsteht kein nennenswertes Gedicht. Wir kennen solche Perioden:in ihnen konventionalisiert sich die Tradition - etwa im Meistersang, mit Ausnahmen in der Liebesund Naturlyrik nach der Mitte des 19. Jahrhunderts -, oder es instrumentalisiert sich die Form - etwa im didaktischen Gedicht des 18. und im politischen des 19. Jahrhunderts. Lyrik erscheint dann als rhetorischer Gestus, der das Platte cachieren 
oder das Postulierte plausibel und eingängig machen soll. Man muß sich klar machen, daß von 100 Gedichten 99, ja von 1000 wahrscheinlich 998 von dieser Art sind, daß aber die Geschichte der Lyrik von den zwei Gedichten pro 1000 lebt. Ein solcher statistischer Hinweis sagt freilich noch nicht allzuviel. Wichtiger ist schon die Frage danach, warum wir z.B. zwischen 1720 und 1770 vielleicht 10, zwischen 1770 und 1830 aber sicher 100 bedeutende Gedichte verzeichnen, obwohl wir in den jeweiligen Jahrzehnten relativ ebenso viele, sagen wir zeitgenössisch : Gedichtmacher haben. Wie also kommt es zu Qualität ? Die gängige Antwort früherer Jahrhunderte ist die gewesen, das Genie - was sicherlich im 17. Jahrhundert nicht einfach das gleiche meint wie am Ende des 18. Jahrhunderts - das Genie habe sich seltener oder habe sich häufiger gezeigt. Die Antwort ist keineswegs falsch. Es gibt dieses Phänomen, das wir in den letzten Jahrzehnten wahrscheinlich einfach "mangels Masse», wie wir uns auszudrücken belieben, unbeachtet gelassen haben bzw. das wir glaubten durch IQ-Tests beschreiben zu können. Vermögen wir auch nicht sehr viel Verbindliches über dieses Phänomen zu sagen, so doch immerhin in bezug auf die Kunst dies : Geniales zeigt sich nur dort, wo eine bestimmte Relation zwischen dem Abarbeiten an der Tradition und dem spontanen Entwurf erreicht wird. So ist der kommune Satz zu verstehen, große Künstler zeichneten sich durch $10 \%$ Genie und $90 \%$ Fleiß aus. Der Fleiß bewährt sich aber nicht nur durch jenes Abarbeiten an der Tradition, sondern auch durch die Herstellung der genannten Relation selbst, genauer : durch die bis zum Kalkül gehende, höchst bewußte verändernde Aufnahme der Tradition in Verbindung mit dem Entwurf, der sich an dieser mißt und prüft. Das bedeutet : Zeiten der Epigonalität wie Zeiten der bloßen Neuigkeit haben ihr Gemeinsames darin, daß es faule Zeiten sind im doppelten Sinn. Wer nur bestätigen, wer nur anders sein will, macht es sich bequem und trägt zur Stagnation bei, sei es als dumpfes Dahinfaulen, sei es als fauler Zauber. -

2 Die Nachkriegszeit ist, auch lyrisch, relativ gut übersehbar. Wir kennen die Etappen: Trümmerlyrik, Traditionalismus v.a. im Naturgedicht, konkrete Poesie, politisches Gedicht, Alltagslyrik, Postmoderne. Die großen Überlebenden, Benn und Brecht, sind ihre eigenen Epigonen geworden. Sie schreiben in ihrem Ton neue Gedichte wie Benns Lebewohl oder Worte oder Melancholie und Brechts Der Blumengarten oder Ach nur der flüchtige Blick. Aber sie schreiben auch bereits Verse wie «Berlin in Klammern und Banden, / sechs Meilen eng die Town / und keine Klipper landen /, wenn so die Nebel braun... " Oder: "Und das Ulbrichtstadion / Und der Erste Mai / Und wenn Ernst Busch singt - / Wärt ihr nur dabei ! »²

3 Celan, Nelly Sachs, Ernst Meister - in einigem Abstand Eich, Kaschnitz, Bachmann, Hüchel, Bobrowski, die alle erst nach dem Krieg bekannt werden, sind wichtige, sind merkenswerte Namen. Und namentlich von den drei ersten kommen die Gedichte her, an die man sich erinnern wird aus den letzten vierzig Jahren, falls man sich noch erinnern wird. Aber niemand wird sagen, diese vierzig, fünfundvierzig Jahre seien, was deutsche Gedichte angeht, mit den vierzig Jahren zwischen 1890 und 1930 vergleichbar, in denen jene vier oder fünf Gedichte, die nach Benn ausreichen, jeweils beigetragen wurden : von George, Hofmannsthal, Else Lasker-Schüler, Rilke, Trakl, Heym, Loerke, Benn, Brecht, nicht zu reden von denen, deren Äquivalent (in geringerer Zahl) wir vielleicht in den letzten vierzig Jahren gehabt haben. Nein, es hat sich im Produktiven etwas geändert und wenn die achtziger Jahre ein Resümee dessen sind, was vorausging, so können sie unter Umständen Auskunft darüber geben, was sich geändert hat. 
4 Ich stütze mich bei dieser Frage auf zwei Anthologien, die sich selbst als solche der achtziger Jahre verstehen, obwohl sie schon 1987 bzw.1988 erschienen sind, also mit jener Atemlosigkeit, die vielleicht ein erstes Indiz der heutigen literarischen Produktionsweise im ganzen, also auch der lyrischen ist. In Michael Brauns und Hans Thills Anthologie Punktzeit (im folgenden zitiert « Braun/Thill») und in Hans Benders Was sind das für Zeiten (im folgenden zitiert «Bender ») sind insgesamt 176 Autoren versammelt, davon 106 nur in Benders Sammlung, 14 nur in Punktzeit, 56 also in beiden. In Hermann Kortes Überblick Geschichte der deutschen Lyrik von 1989 werden 84 dieser Autoren genannt. Bei Korte finde ich auch 14 Lyriker, die in den beiden Anthologien nicht vertreten sind, darunter bekannte Namen wie Brasch, Biermann, Handke, Wondratschek. Ich vermute, daß dies nicht auf einer ausschließenden Entscheidung der Herausgeber beruht, sondern auf urheberrechtlichen Problemen oder auf der Weigerung der Autoren, sich zu beteiligen. Wie immer: man kann annehmen, daß der größte Teil der nennenswerteren Gedichtschreiber, die in den achtziger Jahren publiziert haben, in den beiden Bänden vertreten ist.

5 Eine populäre Sammlung wie Maximilian Berns Neue deutsche Lyrik von 1924, die immerhin einen Zeitraum von ca. 40 Jahren dokumentiert, enthielt nur 147 Autoren, davon waren mindestens 15 längst vor 1880 als dem imaginären terminus post des Bandes bekannt gewesen. In dieser Sammlung stehen, allerdings einigermaßen verloren, ein paar große Gedichte von Mörike, G. Keller, Nietzsche. Ansonsten ist das ganze konventionelle Mittelmaß jener Zeit versammelt: Der allergrößte Teil dieser Gedichte ist strophisch, der allergrößte gereimt. Die Konventionalität ist so bestimmend, daß ein Setzer Effekt machen kann dadurch, daß er in einem paarreimig gedichteten Text den Reim so aufhebt : «Der Herbstwind nahm ihr alle Blätter weg / nur eines blieb ; es weht, verwelkt, verdorrt... » ${ }^{3}$

6 In den Anthologien zu den achtziger Jahren gibt es relativ wenige strophische und ganz wenige gereimte Gedichte. Gezählt habe ich neun «reimende » Lyriker. Die große Mehrheit der heutigen deutsch schreibenden Gedichtautoren scheint ganz fern der Tradition zu arbeiten. Das mindeste ist ein reimloser vers libre, wobei tendenziell von einem ausgeprägten Rhythmus relativ selten, von Prosafügung relativ oft $\mathrm{zu}$ sprechen ist.

7 Hinsichtlich der modernen Lyrik, also der der Zeit von etwa 1890 bis 1930, spricht Hartwig Schultz von einem "harten, gestauten "4, auch vom "gebrochene[n] Rhythmus $»^{5}$ als Signalen dafür, daß das "lyrische Ineinander von Subjekt und Objekt nicht mehr möglich» sei ${ }^{6}$. Doch weist Schultz darauf hin, daß auch die «Formen der modernen Lyrik» "ihre Vorbilder haben $»^{7}$, und daß es nur um eine Bevorzugung bestimmter, längst bekannter Mittel gehe, also eben um ein Abarbeiten an der Tradition.

8 Anders ist das, was etwa seit Ende der sechziger Jahre als Alltagslyrik auftritt. Das ist ein Begriff, der sowohl die Veränderung des Thematischen, nämlich zum Trivialen hin, wie die Veränderung der Form, nämlich zur Umgangssprache und damit zur Prosa hin, anzeigen soll. Nun kennen wir Alltagsthemen bereits in Gedichten des Naturalismus, z.B. bei Holz, oder in denen des Expressionismus, und auch der Gebrauch der Umgangssprache im Gedicht ist nichts Neues. Doch geht es wohl im wesentlichen um die ästhetische und poetologische Perspektivierung dieser Lyrik. So wird z.B. über R. D. Brinkmanns Gedichte gesagt, sie seien ein « Versuch, in Lyrik an die photographischen Medien anzuknüpfen $»^{8}$. Hier wird etwas postuliert, was in der Erzählprosa schon seit 
dem Ende der zwanziger Jahre durchgesetzt zu sein scheint : die Vorbildfunktion eines anderen Mediums für die Literatur und die Identifizierung oder doch Analogisierung von prosaischer Alltagssprache mit Fotografie und Film.

Das aber bedeutet nichts anderes als die Ignorierung der Geschichte der modernen Literatur im allgemeinen und im besonderen der Lyrik. Mailarmés Bemerkung, daß die «Würdigung des Wortes bedeutet, das Eigentlichste und Innerste unseres Geschlechts zum Ruhme führen in seiner Blüte, der Sprache ${ }^{9}$, scheint gar nicht mehr zur Kenntnis genommen, geschweige denn bedacht worden zu sein. Es geht dabei nicht einmal darum, ob die Linie Maliarmés als poésie pure weiterverfolgt wird oder nicht, es geht um die unhintergehbare Einsicht, die gerade in der Lyrik fundamental ist und sich seit Baudelaire, Rimbaud und eben Mallarmé gegen den positivistischen Realismus durchsetzt, daß ein Gedicht nämlich aus Worten und Sätzen und nicht aus der Mimesis von Gefühlen und Vorstellungen von Dingen besteht. Letzteres zu behaupten ist jenes Mißverständnis, das zwar die Sprache der Wissenschaften und des Journalismus bis heute bestimmt, aber nur solange, als das Sprachdenken seit dem 18. Jahrhundert verdrängt wird.

Es fällt auf, daß das Alltagsgedicht oder umfassender die Tendenz zur Beschreibung auch in der Lyrik der achtziger Jahre noch vertreten ist, wenn auch mit z.T. erheblichen Modifikationen. Doch registriere ich in den beiden Anthologien eine Gruppe von vierzig Lyrikern (von 176), die, wenn auch nicht ausschließlich, sich zu Beschreibungen verstehen.

11 "Du siehst den Sammler in seiner Grotte », beginnt das Gedicht Der Sammler von Cyrus Atabay. Es nennt die Sammelgegenstände, es beschreibt ihre Farben, das Berührungsempfinden des Sammlers. Und es endet :

Seine Leidenschaft ist ein Durst,

den nur der Anblick der Dinge stillt

(und aufs neue weckt),

sie sind der Zusammenhang,

der sein Leben verbindet. (Bender, S. 76)

Die Beschreibung beruft sich auf das Visuelle, und zwar das des lyrischen Sprechers wie das des Portraitierten. Das Gedicht behauptet, es teile optische Eindrücke mit.

Interessant ist nun, wie sehr auf der einen Seite lyrische Autoren sich weiterhin von der Auffassung bestimmen lassen, es gehe im Gedicht um die Beschreibung optischer oder akustischer Phänomene, wie sie sich aber fast nie mit dieser Beschreibung begnügen. Hans-Jürgen Heises Ein türkisches Café repräsentiert noch am stärksten die kurze Tradition des Alltagsgedichts.

Ein türkisches Café

Die Fliegen auf meinem Handrücken

sind allein noch kein Orient

doch sie gehören dazu

Ein türkisches Café/ich bin

der einzige Gast

Die grüne Tür

führt auf den Abort

die blaue in die Küche 
auch da bloß Fliegen

Und der Teekessel ein besonders

großer Brummer

summt (Bender, S.93)

Alltagslyrik als Beschreibung zeigt sich insbesondere im Mittelteil, wobei die Wiederholung der Überschrift eine redundante Protokollgenauigkeit repräsentiert : die Fliegen, dreimal evoziert, sind dreimal Verschiedenes: im Mittelteil v.a. Teil der Beschreibung, durch das Adverb semantisch an das Vorausgehende gebunden. Das aber ist eine bescheiden witzige Bemerkung, die auch in jedem Reisefeuilleton vorkommen könnte. Der Schluß dann ein ebenso bescheidener Wortwitz, wobei die Beziehung zwischen «Fliegen» und «Teekessel» nur über das 'Brummen' und 'Summen' geht, was das Thema Ein türkisches Café nicht intensiver, interessanter, überraschender macht. Immerhin zeigt sich, daß jenes mißverstandene 'Fotografische' des Alltagsgedichts sich mit anderen Sageweisen verbindet, wobei allerdings nur die letzten drei Zeilen in ihrer zunehmenden Verkürzung eine rhythmische Relation zu Lyrik haben.

15 Eine Verbindung scheint besonders auffallend zu sein. Ich nenne sie die Verbindung von Beschreibung und Reminiszenz. Dabei geht es um die Evokation von Vergangenem oder um Erinnertes, das dann als Phänomenales benannt oder beschrieben wird.

Ursula Krechel nennt ein Gedicht Erster Februar und läßt es so beginnen : "Was von einem Winternachmittag bleibt: / ...» (Bender, S. 187) Das registriert sie, fügt eine Reflexion und zwei weitere Phänomennamen an und endet mit: «Genügt, was nicht genügt, was bleibt?» (Bender, S. 188) Man bemerkt sofort das etwas baukastenhaft Montierte. Die Beschreibung wird mittels eines Satzes zur Reminiszenz, die Reminiszenz im «bleibt» führt dank diesem Verb zur Reflexion. Auf der einen Seite wird in dem Gedicht begriffen, daß die (reihende) Beschreibung noch kein Gedicht ergibt, insofern sie bloß Protokoll wäre ; andererseits aber verändert die Ankopplung einer resümierenden Formel und zweier Reflexionen den Text noch nicht grundsätzlich. Es sind eher Materialien zu einem Gedicht als dieses selbst.

In Ulrich Schachts Versen Am Mechower See wird Beschreibung ständig mit Reminiszenzen verbunden : ein Haus, eine Straße, Natur verknüpft mit dem, was vor « zerschnitten »- also Trennung durch Politik - war und galt. Worte und Wendungen wie jenes durchziehen den Text und verändern dadurch Beschreibung und Protokoll. Dazu kommen Rhythmisierungen, Akzentuierungen als genuin Lyrisches, das die Unterbrechung, die Trennung mit evoziert.

\author{
Hier kommt \\ kein Mensch den Apfel \\ zu pflücken die Pflaume \\ die Birne zu fischen \\ den Fisch. Nur manchmal \\ fährt übers Wasser ein Boot :
}

[...] (Bender, S. 204)

18 Aber so deutlich akzentuiert durch Endstellung "Apfel » und «Pflaume », durch Anfangsstellung «Birne » und "Fisch » werden, so unbefriedigend muß der teilweise Verzicht auf Interpunktion sein, wenn eine Zeile entsteht wie : « die Birne zu fischen ». Und ganz unnötig plakativ folgt der zitierten Strophe diese 
An Bord

von allen guten Geistern

verlassen : Behelmte. Gewehre

im Anschlag. Sie jagen

den Fisch der ihre

eisernen Netze durchbricht (Bender, S. 204)

Nach dem diesen Zeilen unmittelbar vorausgehenden «fährt übers Wasser ein Boot » könnte direkt angeschlossen werden : «Sie jagen / den Fisch der ihre / eisernen Netze durchbricht ».

Man erfährt mit und an einem solchen Gedicht sehr deutlich die Situation der deutschen Lyrik in den achtziger Jahren. Es bleibt die Bindung an die Alltagsphänomene und ihre Beschreibung. Hinzu kommt die Reminiszenz, die jene als etwas erscheinen läßt, das - hier durch Politik - verloren, abgetrennt ist. Es wird begriffen, daß beides - Beschreibung und Reminiszenz - eine Konstruktion literarischer Art erfordert. Doch stehen auch noch nebeneinander : die Prosa von Feststellungen, aus Reminiszenzen hervorgehend, meinungshafte Bewertungen, schließlich eine Sentenz: «...aber / nirgends ist Nähe / so fern ». Die literarische Konstruktion wird nur teilweise dank Rhythmus, Akzent, Zeilenbau etc. zur lyrischen Konstruktion.

21 Vielfach wird auch noch festgehalten an der seit den sechziger Jahren gängigen improvisatorischen Geste, die das Graphische des Zeilenbruchs, den (teilweisen) Verzicht auf Interpunktion als ausreichendes Signal für das zeitgenössische Gedicht gewertet wissen will. Aber diese improvisatorische Geste verrät nur einen Mangel an Fleiß. Dies fällt immer wieder auf: Der Versuch, sich von der aus einem grundsätzlichen Mißverständnis entstandenen Position, die ein Tiefpunkt lyrischer Produktion war, zu entfernen, aber dennoch nicht « die lässige Attitüde », « die Mischung aus Schlamperei und Prätention », die « neue Formlosigkeit » jener Jahre, von der Harald Hartung schon 1975 sprach $^{10}$, aufzugeben. -

Thematisch verselbständigt sich Reminiszenz in zahlreichen Gedichten, intensiviert sich oft zur Melancholie, zur Klage und sogar zum Pathos. In diesem Zusammenhang erinnert sich das Gedicht wieder der Elegie.

23 Annemarie Zornacks Reminiszenzen reihendes Gedicht lauter augenblicke (Bender, S. 105) versucht das für die Epoche seit den dreißiger Jahren charakteristische Nebeneinander von Alltäglichem als Normalem und Katastrophalem in Punktualitäten zu erfassen. Aus dieser Sicht der Reminiszenz ist zweierlei zu entwickeln: das vom Subjekt verinnerlichte Gedenken des politisch-historisch Vergangenen, aber Weiterwirkenden und die persönliche Erinnerung, die fast immer an das Vorausliegende des eigenen Todes rührt.

24 Yaak Karsunke wagt in der ersten seiner lektionen die Komik und das fast von Anfang an Falsche der sich gemeinhin nur affirmierenden Achtundsechziger erinnernd $\mathrm{zu}$ benennen.

Ulla Hahns Erinnerungsgedicht Gertrud Kolmar (Bender, S. 180), das ein gleichnamiges Bobrowskis aufnimmt, sucht die Lektüre der Briefe der Gertrud K., die selbst auf Erinnerungen sich beziehen, $\mathrm{zu}$ einer Mahnung an die Nachgeborenen $\mathrm{zu}$ konzentrieren.

Ganz persönliche Totenerinnerung ist dagegen Rolf Häufs' Augenblick im Juli : «Unter Bäumen naß von Sommerregen / Gedenke ich meiner toten Freunde » (Bender, S. 115). Intensiver ist die bei Richard Wagner dadurch, daß durch die mehrfache Aufnahme des 
Worts « Erinnerung » und durch die Präzisierung dessen, was Erinnerung je und je ist, das Gedicht eine innere Struktur erhält und nicht bloß Notat von Reminiszenzen bleibt.

Die Erinnerung an Begräbnisse ist die Erinnerung an Begräbnisse auf dem Lande.

Die Erinnerung an die Eltern ist die Erinnerung an einen Sonntagsmittagstisch mit

weißem Tischtuch.

Die Erinnerung an die Jugend ist die Erinnerung an einen Rotweinfleck

auf einem weißen Tischtuch.

Ein Pferdewagen mit schwarzem Tuch und dunklem Holz.

Weiße Kniestrümpfe.

Die weiße Kommunionskerze mit der Schleife auf dem Foto.

Ich, blond und dick.

Die fünfziger Jahre aus Tuch.

Die Großeltern, die auf den Lieschen liegen und sich nicht rühren.

Sonst rascheln die Lieschen.

Sonst rührt sich der Mais. (Bender, S. 210)

Eigentümlich ist, wie sich die Reminiszenz im Gedicht der achtziger Jahre zur Melancholie von Wiederholung und Ende steigert. Eigentümlich nicht darum, weil es sich dabei um ein neues lyrisches Sujet handelte, vielmehr ist es ja eines, das seit der Romantik immer wieder erscheint und z.B. bei Benn einen prominenten Platz einnimmt. Doch scheint es für eine Generation, die sich mit nichts anderem als dem gesellschaftlich Relevanten beschäftigen wollte, eine unerhörte Neuentdeckung zu sein.

Das gilt weniger wohl für einen älteren Autor wie Karl Krolow, der mit dem Gedicht In den achtziger Jahren eher anknüpft an Erfahrungen der Nachkriegszeit und damit zu Beginn der Achtziger diese an jene ankoppelt; auch mit vierzeiligen Strophen zu dreihebigen Versen an deren Tradition.

Die sehr viel persönlichere Melancholie von Michael Krügers Elegie weist mit diesem Titel, weist mit sechszeiliger Strophik ebenfalls auf Tradition. Und auch die metaphorische Durchdringung der Erfahrung des väterlichen Todes ist sehr bewußter Traditionsbezug. Die letzte Strophe lautet :

Endlich Erde. Endlich

der Sand in Berlin, hier darfst du

sterben. Schlägst dir den Tod

wie einen Mantel um das Aschenherz -

und gibst dich frei und schweigst :

weil jede Antwort eine Frage wäre. (Bender, S. 163)

Dreihebigkeit (mit Abweichungen), Wiederholung, metaphorische Wendung, Sentenz : wir sind inzwischen weit weg von der improvisatorischen Geste des Alltagsgedichts. Aber sind wir weiter? Es ist, als würden nun wieder die vergangenen, die kunstvolleren Prägungen eingeübt.

Bei einem älteren Lyriker wie Heinz Piontek erscheint diese Wendung zur Tradition nicht nur als Aufnahme der Elegie, sondern als Aufnahme von Walthers Elegie, als eine Art Cento aus direkten Zitaten («Ich hör ein Wasser summen. »; « Ich sehe schwarze Galle mitten in dem Honig schweben. »; «Und was ich wähnte, daß es wirklich wäre, ist es was?»); Zitatanspielungen aus anderen Walther-Gedichten («Der Friede friert. Auf meiner Straße wildert Gewalt. » = " gewalt vert ûf der strâze : / fride unde reht sint sêre wunt. » "Wie saß ich leicht auf einem Stein im Gras » = "Ich saz ûf eime steine... », Bender, S. 62), aber auch aus Scheinzitaten. 

Gedicht vom Lehen und antwortet darauf mit vielen deutlichen und versteckten Anspielungen : Statt des anfänglichen jubelnden "Ich hân mîn lêhen, al die werlt, ich hân mîn lêhen." bei Walther hier das resignative Resümee: "Das Lehen, das ich brauch, wird nicht vergeben.» (Bender, S. 140) ; statt der dankbaren Bestätigung « der edel künec, der milte künec hat mich beraten... » das ironische Lob « Partei mein Fürst : sie hat uns alles gegeben / Und alles ist noch nicht das Leben » (Bender, S. 140). Sehr deutlich ist in beiden Fällen die Aufnahme, die Wiederaufnahme der literarischen Tradition, die doch über Jahrzehnte als ganz problematisch galt. Doch haben die beiden lyrischen Protagonisten der Nachkriegszeit - Brecht und Benn - natürlich ständig mehr oder minder kenntlich nachgewirkt.

tas nie Peter Rühmkorf, der sich vor allem um eine Synthese von Zynismus und Melancholie, ja von Zynismus und Pathos bemüht hat, immer wieder auch mit Reminiszenzen wie in dem Gedicht Laß leuchten !: " Weißt du noch wie du noch Kletten im Haar, / Knöpfe in der Kollekte... / als das Leben anfänglich war / und nach weiterem schmeckte?» (Bender, S. 83) Erstaunlich ist, wie gewissermaßen lizensiert durch den Zynismus Pathos sich vorwagt, etwa in dieser Strophe von Diese vorüberrauschende blaue... :

Frage nicht frag und ergib und besinn dich, ahnungsvoll gegen die Sterne gekehrt ;

solang der Atem noch aus dir und in dich

schwerelos fährt... (Bender, S. 84)

Oder als Abschluß des Gedichts Alles dunkel alles trübe:

Alles klar soweit - noch Fragen?

Letzter Gruß : Niewiederkehr !

Richtige Verliebte tragen

Paradeiser vor sich her. (Bender, S. 85)

Bemerkenswert bleibt natürlich auch das formale Traditions Wagnis von daktylischen Vierhebern mit dem Zweiheber am Schluß, von auf taktlosen Vierhebern der sogenannten Volksliedstrophe, gereimt und sehr eingängig. Selbstverständlich fragt man nach der Legitimiertheit solchen Verfahrens, da doch diese Formen längst zum Schlager abgewandert sind, einer kommerziellen Angelegenheit also ganz und gar, die nur dann einen Augenblick aufhorchen macht, wenn z.B. ein sogenannter Liedermacher bei braver Beibehaltung der tradierten Formen ständig gegen jede Prosodie absurde Akzentuierungen setzt und natürlich auf Anfragen erklären würde, dies sei seine persönliche Note. Gerät nicht Rühmkorf in die Nähe eines zwar hochgestochenen, aber nichtsdestoweniger beiläufigen Tralalas Ist Alexandrinertum angängig? Das ist keine leicht zu beantwortende Frage, zumal ja die Geibelei des vorigen Jahrhunderts die Geschichte der Lyrik schwer belastet und dem Verständnis für Lyrik unendlich geschadet hat. Aber man muß die veränderte Situation bedenken. Nicht mehr Metrisches und Gereimtes repräsentiert heute das ganz und gar Durchschnittliche, sondern eben die mit «improvisatorischer Geste » vorgetragenen Notate, deren Zeilenbruch nur zu häufig die Chuzpe ist, eine absolute Banalität als Gedicht auszugeben.

Rühmkorfs Gedichte parodieren die Tradition, aber sie erinnern sie auch für jene, die längst meinen, Literatur sei so etwas wie Verschränkung von Feuilleton, Reportage und Kommentar. Gewiß ist seine Haltung allein nicht genug, so wenig wie die Pathos Haltung genug ist, die wir z.B. bei Walter Helmut Fritz, bei Christoph Meckel, bei Bernd 
Nellessen finden. Doch bedeutet sie Widerstand gegen die Unverbindlichkeit und Beiläufigkeit des Gängigen, das in so vielen Gedichten der achtziger Jahren hörbar wird. Denn Konventionalisierung des Unkonventionellen ist ja um keinen Deut wichtiger als die Herz-Schmerz-und Kreuzreim-Poesie zwischen 1860 und 1910. Und nicht nur das Alltagsnotat, auch die Manierismen bis hin zu einer kunststoffanalogen Design Produktion sind nicht nur langweilig, sondern zeitraubend lästig.

Sprengung von konformem Lexikon und konventioneller Syntax, freie Assoziation, Häufung von Paradoxa etc. sind natürlich noch keine energische und eigentümliche Wendung, sondern nur Wiederholung von Surrealismus und Dadaismus. Die selbst aber waren ja keine traditionsfähigen lyrischen Ausdrucksweisen, sondern grenzbestimmende, transzendentale, die einen Schlußstrich oder Anfangspunkt markierten und nicht fortsetzbar waren, so wenig wie ein Ready-made von Duchamps, das ja immer nur reproduziert werden kann.

Am passabelsten sind davon noch Texte, die mit komischen Effekten arbeiten wie ein Frühlingsgedicht von Jan Koneffke (Bender, S. 239 f.). Aber hatten wir auch das nicht im deutschen Expressionismus : bei van Hoddis und Lichtenstein, die das Gedicht zitiert, schon präziser? Und was kann Oskar Pastiors quars vodis (Braun/Thill, S. 148) uns an Sprachparodie, Sprachabsurdität über das hinaus bieten, das wir bei den Dadaisten, aber auch schon bei Scheerbart und in Morgensterns Großem Lalula hatten? Dies alles sind Traditionalismen wider Willen, die nur darum nicht sofort als solche auffallen, weil sie mit erheblicher historischer Unkenntnis rechnen können. Warum aber muß es in diesen Versuchen bei der mehr oder minder geschickten Wiederholung bleiben ? Einfach darum, weil das Zusammen-bzw. Gegeneinanderspiel von Rhythmus und Gedanke nicht mehr stattfindet.

Gerade wenn alles zu gehen scheint, geht nichts mehr, tritt, was als so eigenwillige Bewegung verstanden werden will, auf der Stelle. Der durchgängige Verzicht auf metrisch orientierten Rhythmus oder doch auf einen nachvollziehbaren Gesamtrhythmus des ganzen Textes, ungewöhnliche Substantiv-AdjektivVerbindungen, Häufung kühner Metaphern, zur Regel gewordene syntaktische Abweichungen wie Soloecismen etc. lassen keine Nuancierungen zu, sie wirken als bloße Kumulation des Irregulären und nicht mehr als spezifisch andere Texte, die das konventionelle Sprechen bestreiten, verändern oder als dieses bewußt machen, sondern sie erscheinen als das Beliebige, als Absonderlichkeiten, die ohne weiteres hingenommen werden können. Das ist kein neues Phänomen; wir treffen es schon in der Romantik, z.B. bei Tieck, und es ist immer von autistischer Übersteigerung und damit von Spannungsverlust gegenüber der Konvention bestimmt.

Das Antidot dazu könnte die Tendenz zur Simplizität, ja zum Lakonismus sein, die zunächst bei älteren Autoren wie Erich Arendt, Rose Ausländer, Erich Fried, Peter Hartling, Hermann Lenz auffällt, aber dann auch bei jüngeren Lyrikern wie Bulla, Hensel, Rheinsberg begegnet, manchmal verschwistert mit einer gewissen Preziösität. Von Hans Georg Bulla heißt ein ganz unpreziöses Gedicht Der Vater :

Das ist sein Stuhl,

sein Sessel.

Da ist er gesessen.

Das Leder wurde brüchig unter seinem Körper, um den habe ich keinmal 
meinen Arm gelegt.

Dunkle Flecke auf der Lehne, da lagen seine Hände mittags und oben der Kopf in den Schlaf gelegt.

Auf diese Seite ist er schräg gerutscht. So fand ich ihn. (Bender, S. 193)

Mitten in der protokollierenden Kargheit melden sich Erinnerung und Versagen. Einfache Worte, einfache Sätze stellen auf lakonische Weise eine Grundfigur her, die im Titel aufgerufen wird. Das Verdienst, das solch einem Text zukommt, ist, daß er aufmerksam macht auf sprachliche Grundfiguren, daß er Worten wie "Stuhl, Sessel, Leder, Lehne », Worten wie « Körper, Arm, Hände, Kopf » Aufmerksamkeit verschafft und ihrer Beziehung zueinander, die durch die Verbformen "gesessen, gelegt, gerutscht » hergestellt wird.

Es deutet vieles darauf hin, daß nur mit diesem Lakonismus im Augenblick sinnvoll zu arbeiten ist, weil er sich an den einfachen Sprachbeständen als Elementen der Lyrik orientiert. Nicht das nichtssagende Alltagsgedicht, dem irgendein ornamentales Element appliziert wird, nicht der zur Privatheit neigende Reminiszenz-Text, schon gar nicht die dadaistisch-surrealistischen Repetitionen können die generelle Tendenz zum Beliebigen überwinden helfen, sondern die Konzentration auf Sprachelemente, soweit sie denn mehr als nur Klang, aber auch mehr als nur Zeichen sind.

Formal zeigen sich Simplizität und Lakonismus v.a. im Epigramm oder besser im Epigrammatischen. Das ist, wie wir aus der Geschichte der Lyrik wissen, nie eine zentrale Erscheinung der Lyrik gewesen, aber eine, die als Einübung in den lyrischen Sprachgebrauch eine große Rolle in langer Geschichte gespielt hat. Im 17. und 18. Jahrhundert heißt sie im Deutschen « Sinngedicht » und betont damit einen Anspruch, der nach der Seite des Witzes am ehesten bei Logau und Lessing, nach der Seite der Mystik bei Angelus Silesius eingelöst wird. Nie geht es bei den relevanten

Beispielen der Gattung allein um die Pointe, die auf den Vers gebracht wird, es geht vielmehr darum, diese Pointe durch Lexikon, Syntax, Vers so vorzubereiten, daß sie so etwas wie deren Frucht ist.

Was in den achtziger Jahren an Epigrammatischem vorgelegt wird, ist zwar manchmal zu preziös, manchmal auch zu platt und simpel. Aber manchmal gibt es eine Ahnung von dem, was Lyrik wieder sein könnte. In einem autorkritischen Gedicht Woraus schöpft der Poet? versucht Walter Gröner einfache Maximen durch einfache Rhythmen, in einfachen Metaphern zu fassen. Dabei mißlingt eine Zeile auf kuriose Weise, weil die simplizistische Attitüde der siebziger Jahre für Einfachheit gehalten wird.

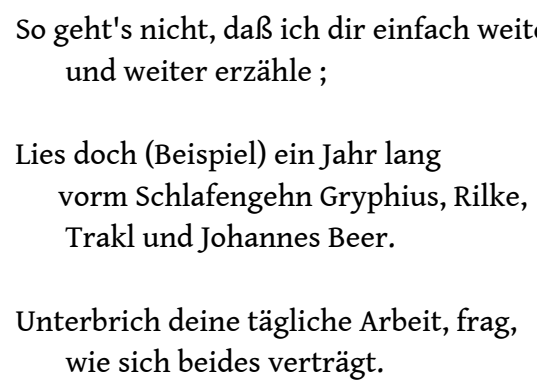


Falle in Löcher. Wende dich ab von der schönen Natur.

Stehe manchmal belämmert umher.

Wenn die Schwärze nicht weicht, singe laut ein Sonett aus der Erinnerung, oder es kann auch was Selbstgedichtetes sein.

Bilde Worte. Trage die Worte wie Kohlen in jedes Gelände hinaus.

Bevor es ganz unerträglich brennt, schmeiß sie wohin.

Sage zum Dichter : da liegen Brocken von mir.

Werde nicht gleich wieder frech. (Bender, S. 201 f.)

Dennoch:Daß wieder von einfachen Worten die Rede ist, von der Arbeit mit Worten, von aufmerksamer Lektüre, von irritierter Bescheidenheit, ist schon ein brauchbarer Anfang.

Peter Hamm macht aus einem Eindruck, dem von fahrendem Zug und Landschaft, ein Epigramm (Speisewage;Bender, S. 130), das durch das überwertige Buch des Lebens allerdings zu komplett wird.

Sehr verwandt ist ein zweites, Zugabteil, mit dem pointierenden Schluß : «Umkehr, Umkehr : / um nichts in der Welt / und um jedes » (Braun/Thill, S. 29.) Lakonisches Pathos, freilich immer schon gefährdet, Predigt zu werden.

Melancholie und Satire verbinden sich schließlich in Reiner Kunzes Gedicht Berühmter Dichter des Westens signiert. Lakonisch der Eindruck am Anfang - und schon kritisch ; lakonisch die Erinnerung - und auch melancholisch. Beides wird am Schluß als unaufhebbarer Widerspruch zwischen dem Buch als Existentiellem und als Ware zugespitzt. Es ist der Widerspruch zwischen der Leistung und dem Versagen der Literatur, das in der Geste des signierenden Dichters erscheint.

Band um band, aufgeschlagen ihm gereicht vom stapel,

reinigt den Schmutztitel er

mit dem vollkommenen gewitter seiner unterschrift

Das halbe leben fast bewachten wir das buch, das wir ihm

vor die feder legen (abdrücke trägt's

eines dielenbretts, unter dem wir es einst

verbargen)

Er sieht's und sieht : vom Stapel nicht -

und schiebt's beiseite

mit dem initial (Bender, S. 107)

Dies sind nur einige Beispiele des epigrammatischen Gedichts, die von solchen des Gedankengedichts und des satirischen Gedichts, ebenfalls oft am Lakonismus orientiert, erweiterbar wären.

Man merkt, es ist hier nichts Großes, nichts entschieden Berührendes aufzuzeigen. Das Beste ist ein Reduktionismus, der die Worte ausstellt und sich nicht vor sie stellt, der vorsichtig mit ihnen umgeht, der sie so zeigt, daß sie schon an sich selbst als Gedanke erscheinen, der das private Gespreiz draußen läßt und auch einen Manierismus, der von gestern ist und nie mehr als Kunsthandwerk. Damit der ekelhafte Medienprosaschleim, der die Welt überzieht, austrockne, muß lyrisch Sprachaskese eingeübt werden. Das Postulat des langen Gedichts war verfehlt. Man gehe vielmehr von der Maxime aus : Prüfe, ob in deinem Gedicht nicht jedes Wort ein Wort zu viel ist. Streiche, wenn nötig, den ganzen Text durch.

Die achtziger Jahre zeigen sich auch in der Lyrik als das, was sie ansonsten waren : betriebsam, geschwätzig, prätentiös und einigermaßen platt. Den Lyrikern ist es, soweit man dies aus den genannten beiden Anthologien schließen kann, noch nicht 
gelungen, gegen das Nichtige des Medienschleims ein Wehr der Worte aufzurichten.

Wenige Monate vor Beginn der achtziger Jahre starb Ernst Meister als der letzte, der unter den Deutschen an diesem Wehr gebaut hat. Mit letzten Gedichten von ihm eröffnet Hans Bender seine Dokumentation. Darunter sind zwei lakonische, auch epigrammatische Gedichte : das eine befaßt sich mit der Beziehung zwischen Wort und Schmerz, Wort und Klarheit, Wort und Ursprung; das andere mit den Worten, die als Namen sich zeigen und darum jenseits des Nichtigen sind. Man braucht nur diese Texte zu hören, um den Abstand zu erfahren selbst zwischen den bemühtesten aus den achtziger Jahren und ihnen. Sie freilich genügen, um unsere fast allein aus Geschwätz und Gewalt bestehenden Jahrzehnte nicht ins Bodenlose fallen zu lassen.

DAS WORT,

Hand in Hand

mit dem Schmerz,

stülpt es um,

so daß

ein gewendetes

wäre

durch deutliches Zeigen?

Klarheit

entsteht, was sonst,

die Seele erleichternd.

Nähe des Ursprungs. (Bender, S. 10)

WAS, vor allem,

weiß ich schon bald

von Namen noch :

Haus... Baum...

Blume... Ach,

denke nicht,

daß deinen Staub

Nichtigsein

quäle. (Bender, S. 10)

\section{NOTES}

1. Gottfried Benn, Bar. In:G. B., Gedichte = Gesammelte Werke, Bd. 3, ed. D. Wellershoff. Wiesbaden 1960. S. 284.

2. Bertolt Brecht, Angebot. In : B. B., Gesammelte Werke, Bd. 10. Frankfurt/M.1967. S. 995.

3. Maximilian Bern [Hrsg.], Neue deutsche Lyrik. Berlin 1924. S.U.

4. Hartwig Schultz, Vom Rhythmus der modernen Lyrik. Parallele Versstrukturen bei Holz, George, Rilke, Brecht und den Expressionisten. München 1970 (Literatur als Kunst. Ed. W. Höllerer.) S. 137. 
5. a.a.O.S. 139

6. a.a.o.

7. a.a.o.S. 143

8. B. Urbe, Lyrik, Fotografie und Massenkultur bei Rolf Dieter Brinkmann. Frankfurt/M. 1985. S.

51. Zit. nach H. Korte, Geschichte der deutschen Lyrik seit 1945. Stuttgart 1989 (Sammlung Metzler, Bd. 250.) S. 149.

9. Hugo Friedrich, Die Struktur der modernen Lyrik. Von Baudelaire bis zur Gegenwart. Hamburg 1956. (Rowohlts deutsche Enzyklopädie.) S. 97.

10. Harald Hartung, Experimentelle Literatur und konkrete Poesie. Göttingen 1975. S. 50,62. Zit. nach H. Korte, Geschichte der deutschen Lyrik, [s. Anm.8.] S. 147.

\section{RÉSUMÉS}

Après les étapes qu'ont constitué la poésie des ruines, le traditionalisme, la poésie concrète, le poème politique, la poésie du quotidien et la post-modernité entre 1945 et 1980, deux anthologies (Braun/Thill, Punktzeit et Bender, Was sind das für Zeiten) présentent comme forme poétique déterminante des années quatre-vingt le poème du quotidien et la description, souvent élargie par la réminiscence. Mais assez souvent apparaissent aussi, de manière assez large et éclectique, un recours au traditionalisme qui va jusqu'à la parodie, ainsi qu'une répétition du surréalisme et du dadaïsme. S'y opposent un laconisme et une tendance au style épigrammatique qui, en tant que modes du réductionisme, sont l'expression d'une ascèse verbale et peuvent ainsi être les plus aptes à lutter contre la verbosité de cette décennie.

Nach den Etappen Trümmerlyrik, Traditionalismus, konkrete Poesie, politisches Gedicht, Alltagslyrik, Postmoderne in der Zeit zwischen 1945 und 1980 zeigen sich in zwei Anthologien (Braun/Thill, Punktzeit; Bender, Was sind das für Zeiten) als bestimmende lyrische Ausdrucksweisen der achtziger Jahre das Alltagsgedicht und die Beschreibung, die oft durch Reminiszenz erweitert wird. Aber auch die Wendung zum Traditionalismus bis zur Parodie, die Wiederholung von Surrealismus und Dadaismus erscheinen häufiger, und zwar durchweg breit und eklektizistisch. Ihnen tritt ein Lakonismus und die Tendenz zum Epigrammatischen entgegen, die als Weisen des Reduktionismus Sprachaskese demonstrieren und so am ehesten der Geschwätzigkeit des Jahrzehnts entgegen- wirken können.

\section{AUTEUR}

HELMUT ARNTZEN

Universität Münster 